\title{
Treatment of allergic conjunctivitis with olopatadine hydrochloride eye drops
}

\author{
Eiichi Uchio \\ Department of Ophthalmology, \\ Fukuoka University School of \\ Medicine, Fukuoka, Japan
}

Correspondence: Eiichi Uchio Department of Ophthalmology, Fukuoka University School of Medicine, 7-45-I Nanakuma, Jonan-ku, Fukuoka 8I4-0I80, Japan

Tel +81928011011

Fax +81 928654445

Email: euchio@fukuoka-u.ac.jp

\begin{abstract}
Olopatadine hydrochloride exerts a wide range of pharmacological actions such as histamine $\mathrm{H}_{1}$ receptor antagonist action, chemical mediator suppressive action, and eosinophil infiltration suppressive action. Olopatadine hydrochloride $0.1 \%$ ophthalmic solution (Patanol ${ }^{\circledR}$ ) was introduced to the market in Japan in October 2006. In a conjunctival allergen challenge (CAC) test, olopatadine hydrochloride $0.1 \%$ ophthalmic solution significantly suppressed ocular itching and hyperemia compared with levocabastine hydrochloride $0.05 \%$ ophthalmic solution, and the number of patients who complained of ocular discomfort was lower in the olopatadine group than in the levocabastine group. Conjunctival cell membrane disruption was observed in vitro in the ketotifen fumarate group, epinastine hydrochloride group, and azelastine hydrochloride group, but not in the olopatadine hydrochloride $0.1 \%$ ophthalmic solution group, which may potentially explain the lower discomfort felt by patients on instillation. Many other studies in humans have revealed the superiority of olopatadine $0.1 \%$ hydrochloride eye drops to several other anti-allergic eye drops. Overseas, olopatadine hydrochloride $0.2 \%$ ophthalmic solution for a once-daily regimen has been marketed under the brand name of Pataday ${ }^{\circledR}$. It is expected that olopatadine hydrochloride ophthalmic solutions may be used in patients with a more severe spectrum of allergic conjunctival diseases, such as vernal keratoconjunctivitis or atopic keratoconjunctivitis, in the near future.
\end{abstract}

Keywords: olopatadine, eye drop, allergic conjunctivitis, anti-histaminergic

\section{Introduction}

The prevalence of allergic conjunctival diseases (ACD) in Japan is estimated to be as high as $15 \%-20 \%$ of the population and is on the rise. Cases of seasonal allergic conjunctivitis (SAC) due to cedar pollen account for a major part of allergic conjunctivitis (AC) in Japan. Cedar pollinosis presents not only with nasal symptoms such as sneezing and rhinorrhea, but also with severe ocular itching. Ocular itching and nasal symptoms adversely affect the quality of life (QoL) of patients. In addition, the recent increase in severity of ACD is raising concerns.

ACD are ocular disorders caused by allergic inflammation of the ocular surface, and include $\mathrm{AC}$, atopic keratoconjunctivitis (AKC), vernal keratoconjunctivitis (VKC), and giant papillary conjunctivitis (GPC). The clinical features of ACD are characterized by their wide variety, and the medical treatment of ACD should be based on the clinical characteristics. AC is defined as ACD in which the conjunctiva shows no proliferative change, and is divided into two subcategories, SAC and perennial allergic conjunctivitis (PAC), according to the presence of seasonal exacerbation. PAC is a less severe ACD, though still uncomfortable for the patient. Signs and symptoms of PAC include itching, hyperemia, and tearing. Mucous discharge is clear and transient. AKC is a bilateral, chronic hypersensitivity disease of the ocular surface seen in association with systemic atopic dermatitis, characterized by lesions of the conjunctiva and cornea that vary in severity. Ocular symptoms include intense itching, photophobia, burning, 
and the sensation of a foreign body. The clinical signs show a wide spectrum, and in the most severe cases, conjunctival scarring with subepithelial fibrosis, fornix foreshortening, symblepharon and corneal ulceration, and neovascularization may occur. Therefore, it is a sight-threatening condition, emerging in the second through the fifth decade of life (Belfort et al 2000). The disease is associated with eczema of the lids or other parts of the body. VKC is a recurrent seasonal disease of childhood, characterized by severe bilateral inflammation of the conjunctiva and by giant papillae of the superior tarsal conjunctiva, gelatinous hypertrophy of the limbus, and keratopathy. It is associated with intense itching, photophobia, and mucous discharge, and is most commonly seen in male patients. However, unlike the severe group of $\mathrm{AKC}$, it tends to resolve spontaneously after several years. The incidence of VKC varies markedly with geographic location, with individuals in Italy, Japan, and other areas of warm climate being more likely to have the disease (Calonge 1999; Ono and Abelson 2005).

For the treatment of ACD, several local ocular drugs, such as antihistamines, anti-allergic agents, and corticosteroids, have been developed as commercially available eye drops recently. Anti-allergic eye drops products which are the basic therapy for ACD are classified into 2 types based on their pharmacological characteristics: drugs that suppress the release of a mediator (eg, disodium cromoglycate) and histamine $\mathrm{H}_{1}$ antagonists. Especially, histamine $\mathrm{H}_{1}$ antagonists are recommended as the first choice of treatment for cases with severe nasal symptoms and itching because prompt symptom relief is expected.

As of 2005, 6 mediator release suppression agents (disodium cromoglycate, amlexanox, pemirolast potassium, tranilast, ibudilast, and acitazanolast hydrate) and 2 histamine $\mathrm{H}_{1}$ antagonists (ketotifen fumarate and levocabastine hydrochloride) are used in commercially available anti-allergic eye drops products in Japan. Although prescriptions of eye drops containing histamine $\mathrm{H}_{1}$ antagonists have recently increased because of their superior rapid effect, only 2 eye drops, ketotifen fumarate $0.05 \%$ and levocabastine hydrochloride $0.025 \%$, were available at that time in Japan; in contrast, more anti-histaminergic eye drops were available worldwide.

Olopatadine hydrochloride, developed by Kyowa Hakko Kogyo Co., Ltd. (Tokyo, Japan), exerts a wide range of pharmacological actions such as histamine $\mathrm{H}_{1}$ receptor antagonist action, chemical mediator suppressive action, tachykinin release inhibitory action, and eosinophil infiltration suppressive action. In Japan, a formulation for oral use that is highly evaluated by physicians and is indicated for allergic rhinitis, urticaria, and itching associated with dermatosis (eczema and dermatitis, prurigo, pruritus cutaneous, psoriasis vulgaris, and erythema exsudativum multiforme) has been marketed since March 2001 (Ohmori et al 2002). Alcon Inc. (Hünenberg, Switzerland) developed an olopatadine hydrochloride ophthalmic solution under license from Kyowa Hakko Kogyo Co., Ltd. Since 1996, the product has been approved in about 90 countries, including the US, and at present it is widely used in clinical practice to treat AC.

In Japan, olopatadine hydrochloride $0.1 \%$ ophthalmic solution was introduced to the market in October 2006 as a third anti-histaminergic eye drop, in addition ketotifen fumarate and levocabastine hydrochloride. This article reviews the pharmacological actions and clinical effects of the new olopatadine hydrochloride ophthalmic solution.

\section{Pharmacological properties}

ACD are mainly caused by type I allergic reactions. When IgE antibodies specific to pollen or house dust are excessively produced, an antigen-antibody reaction is induced, leading to degranulation of mast cells. Then, mast cells release chemical mediators such as histamine, triggering the development of symptoms. Although such allergic reactions involve various chemical mediators, conjunctival symptoms such as ocular itching and conjunctival hyperemia are developed by histaminergic actions mainly through $\mathrm{H}_{1}$ receptors (Knight 1994).

Histamine $\mathrm{H}_{1}$ receptor selectivity of olopatadine hydrochloride was examined using brain homogenates from guinea pigs and rats. Affinity for $\mathrm{H}_{1}, \mathrm{H}_{2}$, and $\mathrm{H}_{3}$ receptors was examined using pyrilamine, tiotidine, and methylhistamine as the ligand, respectively. $\mathrm{K}_{\mathrm{i}}$ (affinity) values of the olopatadine hydrochloride binding to $\mathrm{H}_{1}, \mathrm{H}_{2}$, and $\mathrm{H}_{3}$ receptors were $4.11 \times 10^{-8} \mathrm{M}, 4.34 \times 10^{-5} \mathrm{M}$, and $1.72 \times 10^{-4} \mathrm{M}$, respectively, indicating that selectivity for $\mathrm{H}_{1}$ receptors is about 1000 times that for $\mathrm{H}_{2}$ receptors and 4000 times that for $\mathrm{H}_{3}$ receptors. The selectivity of olopatadine hydrochloride is higher than that of ketotifen fumarate or levocabastine hydrochloride (Sharif et al 1996). Compared with disodium cromoglycate, nedocromil, and pemirolast potassium, only olopatadine hydrochloride suppressed antigen-induced histamine from human conjunctival mast cells in a dose-dependent manner $\left(\mathrm{IC}_{50}: 6.53 \times 10^{-4} \mathrm{M}\right)$ (Yanni et al 1997).

Olopatadine hydrochloride suppressed TNF- $\alpha$ release in vitro from human conjunctival mast cells in a concentrationdependent manner $\left(\mathrm{IC}_{50}: 1.3 \times 10^{-5} \mathrm{M}\right)($ Cook et al 2000), as well as that of interleukin 6 (IL-6) and IL-8 from conjunctival epithelial cells $\left(\mathrm{IC}_{50}: 5.5 \times 10^{-9} \mathrm{M}\right.$ and $1.7 \times 10^{-9} \mathrm{M}$, 
respectively) (Yanni et al 1999). The effects of olopatadine hydrochloride on type I allergy have also been examined in vivo. Guinea pigs were passively sensitized through administration of anti-ovalbumin (OVA) serum into the conjunctival sac, and then olopatadine hydrochloride was instilled into their eyes. Thirty minutes after the administration, its effect on passive anaphylactic reaction was evaluated by intravenously administering solution containing OVA and Evans blue. As a result, $0.001 \%-0.1 \%$ olopatadine hydrochloride showed a dose-dependent suppressive effect, with the $50 \%$ effective dose $\left(\mathrm{ED}_{50}\right)$ being $0.0067 \%$. Moreover, the passive anaphylactic reaction induced in guinea pigs by instillation of the antigen after sensitization with anti-OVA serum was significantly suppressed when $0.001 \%-1.0 \%$ olopatadine hydrochloride was instilled into the eyes 30 minutes before the induction $\left(\mathrm{ED}_{50}: 0.017 \%\right)$. The effect of olopatadine hydrochloride on enhanced vascular permeability induced by histamine in the conjunctiva of guinea pigs ranged between concentrations of $0.00001 \%$ to $1.0 \%$. Olopatadine hydrochloride suppressed the reaction in a concentration-dependent manner. Significant suppression was observed at $0.1 \%$ for 24 hours compared with that in a control group in which physiological saline was instilled into the eyes (Yanni et al 1996).

Olopatadine hydrochloride is considered as a promising dual-action drug with selective and continuous histamine $\mathrm{H}_{1}$ receptor antagonistic action and mast cell stabilization action.

\section{Clinical trials in Japan}

Olopatadine hydrochloride $0.1 \%$ ophthalmic solution is marketed worldwide as Patanol ${ }^{\circledR}$ Ophthalmic Solution $0.1 \%$. In Japan its approved dosage and administration for allergic conjunctivitis is $1-2$ drops qid. At the beginning of its development in Japan, approval for instillation bid at the same dosage was considered for convenience of clinical use. However, in Japan, most anti-allergic eye drops products are used qid, because patients with severe ocular itching caused by cedar pollinosis tend to prefer to instill more than twice per day. Another reason was that the higher frequency of instillations might have a better antigen flushing effect. An open study was conducted to examine the potential increase of adverse reactions with a higher frequency of instillation. The study compared a bid group with a qid group, 44 patients in each, during the cedar-pollen-shedding period using an environmental study protocol. Effects on itching and hyperemia were comparable in both groups; however, the qid group showed slightly lower incidence of adverse drug reactions than the bid group (bid group, 15.9\%; qid group, 9.1\%) (Saiga et al 2006). Based on these results and the aforementioned reasons, the qid regimen was adopted. A double-blind phase III study that involved 247 patients with Japanese cedar pollen AC was conducted using the environmental study protocol mentioned above. The study compared olopatadine hydrochloride $0.1 \%$ ophthalmic solution with ketotifen fumarate $0.05 \%$ ophthalmic solution (a control drug), both at a dose of 2 drops qid for 28 days. Olopatadine hydrochloride $0.1 \%$ eye drops proved to be as effective (ocular itching and conjunctival hyperemia) as ketotifen fumarate $0.05 \%$ eye drops. The incidence of adverse reactions was $4.8 \%$ (6/124 patients) in the olopatadine group and $20.3 \%$ $(25 / 123$ patients $)$ in the ketotifen group $(\mathrm{p}=0.0002$, Fisher's exact test) (Saiga 2006). Thereafter, a long-term study was carried out in 20 patients with $\mathrm{AC}$ in which the study drug was administered at the same dose but for 70 days (Saiga et al 2005). The patients were evaluated every 2 weeks using an ocular itching and conjunctival hyperemia scale. The ocular itching score decreased with time during treatment: $3.55 \pm$ 1.2 (mean $\pm \mathrm{SD}$ ) at baseline, $2.85 \pm 1.5$ at 2 weeks, $2.13 \pm$ 1.4 at 4 weeks, $2.08 \pm 1.7$ at 6 weeks, $1.75 \pm 1.7$ at 8 weeks, and $1.63 \pm 1.7$ at 10 weeks. The conjunctival hyperemia score showed a similar tendency; signs and symptoms markedly improved after the prolonged treatment period. No adverse reaction was observed, demonstrating the efficacy and tolerability of olopatadine hydrochloride $0.1 \%$ eye drops in the long-term treatment.

\section{Clinical evaluation of olopatadine hydrochloride in CAC study}

Conjunctival allergen challenge (CAC) was developed by Abelson et al (1990) to evaluate the efficacy of anti-allergic ophthalmic solutions. CAC facilitates the evaluation, because in patients confirmed to have antigen sensitivity, conjunctival allergic reaction is reproduced by antigen challenge at a standardized exact concentration, and then clinical symptoms are recorded based on evaluation scales described in the protocol. Because it also allows comparison between right and left eyes in the same individual, it is less likely to be affected by variations in individual differences than conventional environmental studies. Olopatadine hydrochloride was also evaluated for usefulness in terms of duration of action, resolution of ocular itching, and comfort upon instillation, using the CAC study scheme.

Abelson and Greiner conducted a double-blind CAC study that involved 68 patients (Abelson and Greiner 2004). Olopatadine hydrochloride $0.1 \%$ eye drops and levocabastine hydrochloride $0.05 \%$ eye drops were randomly assigned to the right and left eyes in an individual, in a double-blind 
manner. After treatment, ocular findings induced by a positive antigen were compared within 1 hour of dosing. Olopatadine hydrochloride $0.1 \%$ ophthalmic solution significantly suppressed ocular itching ( 3 and 10 minutes after antigen induction, $\mathrm{p}<0.001)$ and conjunctival hyperemia $(3,10$, and 20 minutes after antigen induction, $\mathrm{p}<0.0001)$ compared with levocabastine hydrochloride $0.05 \%$ ophthalmic solution. Fewer patients complained of ocular discomfort in the olopatadine group than in the levocabastine group. Berdy et al (2000) compared ketotifen fumarate $0.025 \%$ ophthalmic solution with olopatadine hydrochloride $0.1 \%$ ophthalmic solution in 32 patients. One drop of the respective eye drops was instilled and the eye was challenged with the antigen 12 hours later. Olopatadine hydrochloride significantly reduced the itching score ( 3 , 5 , and 10 minutes after antigen induction, $\mathrm{p}<0.05$ ) and comfort score $(\mathrm{p}<0.05)$ compared with ketotifen fumarate. Thus, these CAC study results showed that the effects of olopatadine hydrochloride $0.1 \%$ ophthalmic solution are observed within a few minutes after antigen challenge and they last for considerably long period.

Ohno et al (2007) conducted a CAC study that involved 20 Japanese (including patients of Japanese descent) patients with ACD (including Japanese cedar pollinosis) to compare olopatadine hydrochloride $0.1 \%$ eye drops with levocabastine hydrochloride $0.025 \%$ eye drops for efficacy and comfort (Ohno et al 2007). Each eye was challenged with the antigen 3.5 hours after any of the two drugs had been instilled, and ocular itching was evaluated using a 5 -grade scale of 0 (absent) to 4 (severe). Olopatadine hydrochloride markedly suppressed ocular itching compared with levocabastine hydrochloride; the mean score was $42 \%$ lower in olopatadine hydrochloride-treated eyes than in levocabastine hydrochloride-treated eyes. The number of responders (subjects with an ocular itching score of 0 after the treatment) was significantly higher in the olopatadine group than in the levocabastine group ( $p<0.05$ ) (Figure 1). In contrast, ocular pain (25\%) and burning sensation (20\%) were observed in the levocabastine group, but no such symptoms were reported within the olopatadine group. Seventy-five percent (15/20) preferred olopatadine hydrochloride $0.1 \%$ eye drops to levocabastine hydrochloride $0.025 \%$ eye drops according to their objective symptoms.

As described above, the results of CAC studies performed both in Japan and other countries have demonstrated the effectiveness and usefulness of olopatadine hydrochloride $0.1 \%$ ophthalmic solution in reducing ocular symptoms in patients with ACD.

\section{Comparison of olopatadine hydrochloride with other eye drops}

Brockman et al (2003) examined cytotoxicity of each antihistamine in a concentration range at which the drug might be present after instillation by measuring lactate dehydrogenase (LDH), which is released from human conjunctival cells after cell membrane disruption. They reported that cell membrane disruption was observed in the ketotifen fumarate $0.025 \%$ group, epinastine hydrochloride $0.05 \%$ group, and azelastine hydrochloride $0.05 \%$ group, but not in the olopatadine hydrochloride $0.1 \%$ ophthalmic solution group, which may explain why patients felt less discomfort on instillation. In Japan, the aforementioned phase III study showed favorable tolerability of olopatadine hydrochloride $0.1 \%$ eye drops; in addition, Sumi et al (2008) compared the effects and satisfaction rating in 17 patients with Japanese cedar pollinosis who had used other anti-allergic eye drops in the previous year (levocabastine hydrochloride ophthalmic solution had been used by 10 patients, ketotifen fumarate ophthalmic solution by 3 patients, others by 2 patients, and unknown drugs by 2 patients). These patients used olopatadine hydrochloride $0.1 \%$ ophthalmic solution after the onset of symptoms, and graded their ocular itching according to the visual analog scale (VAS). The score decreased from 6.59 at baseline to 3.91 at 2 weeks, with statistical significance. The mean difference of the ocular itching score from baseline score was 7.38 with olopatadine hydrochloride $0.1 \%$ ophthalmic solution, which was higher than the score reduction obtained in the previous year (4.63). The level of satisfaction with treatment was investigated in patients who had used the most commonly prescribed levocabastine hydrochloride eye drops in the previous year; $78 \%$ of the patients preferred olopatadine eye drops to levocabastine

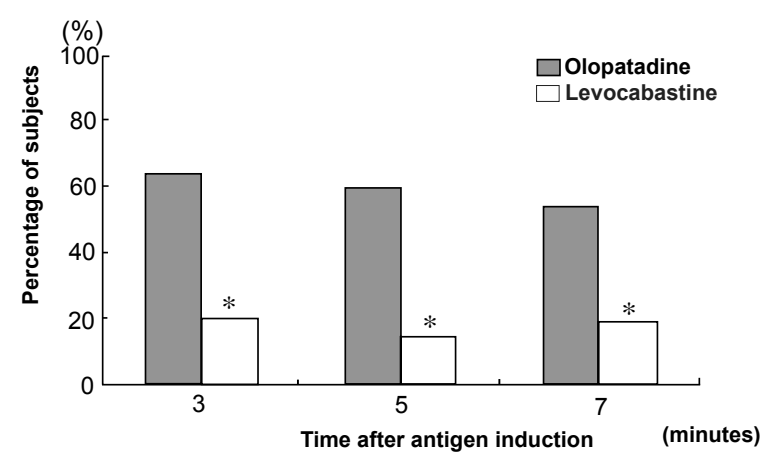

Figure I Percentage of responders: CAC (conjunctival allergen challenge) study comparing olopatadine hydrochloride $0.1 \%$ ophthalmic solution with levocabastine hydrochloride $0.025 \%$ ophthalmic solution. Percentage of responders $(n=20)$. A subject with itching score results of 0 after treatment was defined as a responder. ${ }^{*} \mathrm{p}<0.05$. 
eye drops because of higher therapeutic effects and more comfortable feeling upon instillation.

Overseas, double-blind, controlled studies using an environmental study protocol have reported favorable effects and local tolerability of olopatadine hydrochloride $0.1 \%$ ophthalmic solution. Artal et al (2000) instilled 1 drop of olopatadine hydrochloride $0.1 \%$ ophthalmic solution into either right or left eye, and 1 drop of ketotifen fumarate $0.05 \%$ ophthalmic solution into the contralateral eye in 80 patients to investigate comfort upon instillation. They reported that all patients preferred olopatadine hydrochloride $0.1 \%$ to ketotifen fumarate $0.05 \%$. Aguilar (2000) conducted a randomized double-blind controlled study to compare the efficacy of olopatadine hydrochloride $0.1 \%$ eye drops and ketotifen fumarate $0.05 \%$ eye drops, and tolerability in 80 patients with allergic conjunctivitis. These drugs were instilled bid for 14 days. Olopatadine hydrochloride $0.1 \%$ ophthalmic solution reduced itching as early as 30 minutes after the instillation and a higher percentage of patients reported improvement with olopatadine hydrochloride $0.1 \%$ ophthalmic solution than with ketotifen fumarate $0.05 \%$ ophthalmic solution. Aguilar (2000) also reported that mild reaction of intolerance (stinging) was observed in $23 \%$ of the ketotifen fumarate $0.05 \%$ ophthalmic solution group, but not in the olopatadine hydrochloride $0.1 \%$ ophthalmic solution group. Katelaris et al (2002) conducted a 6-week, multicenter, randomized controlled study to compare the effects of olopatadine hydrochloride $0.1 \%$ ophthalmic solution and disodium cromoglycate $2 \%$ ophthalmic solution on itching and hyperemia in 185 patients with seasonal allergic conjunctivitis. Group I received olopatadine hydrochloride $0.1 \%$ ophthalmic solution bid and placebo bid, while Group II received disodium cromoglycate $2 \%$ ophthalmic solution qid Itching and hyperemia were suppressed in a significantly higher percentage of patients in Group I ( $p<0.05$, vs Group II), and both groups showed favorable tolerability. They also reported that in children less than 11 years old, olopatadine hydrochloride $0.1 \%$ ophthalmic solution seemed to be more tolerable than disodium cromoglycate $2 \%$ ophthalmic solution. It has been reported that olopatadine $0.1 \%$ ophthalmic solution is significantly more effective than epinastine hydrochloride $0.05 \%$ ophthalmic solution in controlling itching, redness, and chemosis associated with allergic conjunctivitis in the CAC model (Lanier et al 2004). Spangler et al (2001) compared the effect of olopatadine hydrochloride $0.1 \%$ ophthalmic solution and azelastine hydrochloride $0.05 \%$ in the CAC model, and found that both treatments were significantly more effective than placebo at reducing itching post challenge; however, olopatadine was significantly more effective than azelastine in reducing itching at 3.5 minutes through 20 minutes post challenge (average mean unit difference of -0.31 ; $<<0.05$ ). In a placebo-controlled, randomized, parallel group, single-center study, both olopatadine $0.1 \%$ and ketorolac $0.5 \%$ ophthalmic solutions were found to be effective in alleviating the clinical signs and symptoms of SAC compared with placebo. However, olopatadine reduces ocular itching significantly more than ketorolac (Yaylali et al 2003).

In contrast, in a 30-day, randomized, double-masked, artificial tear substitute (ATS)-controlled clinical trial, in both active-treatment groups (olopatadine hydrochloride $0.1 \%$ and ketotifen fumarate $0.025 \%$ ophthalmic solutions), the improvements in clinical scores (tearing and itching) were more pronounced compared with those in the ATS group, although the day-30 difference in tearing score between the olopatadine and ATS groups was not statistically significant (Avunduk et al 2005). Ganz et al (2003) carried out a 3-week prospective, randomized, double-masked, parallel-group study to compare ketotifen fumarate $0.025 \%$ ophthalmic solution and olopatadine hydrochloride $0.1 \%$ ophthalmic solution in 66 patients with SAC. The responder rate was higher with ketotifen than with olopatadine on day 5 (72\% vs $54 \%$ for patient assessment, $88 \%$ vs 55\% for investigator assessment) and day 21 (91\% vs 55\%, 94\% vs 42\%). Global efficacy ratings were also higher with ketotifen, and severity scores for hyperemia and itching were significantly lower. Although the reason for these conflicting results is unclear, differences in study design or study population might be the explanation.

\section{Clinical effect of olopatadine $0.2 \%$ eye drops}

Overseas, olopatadine hydrochloride $0.2 \%$ ophthalmic solution for a once-daily regimen has been marketed under the brand name of Pataday ${ }^{\circledR}$. Its effect lasts 24 hours (Vogelson et al 2004). Abelson et al carried out a randomized doubleblind placebo controlled study using a CAC study protocol that involved 23 patients (Abelson et al 2008). Olopatadine hydrochloride $0.2 \%$ ophthalmic solution was instilled once daily into one eye and olopatadine hydrochloride $0.1 \%$ ophthalmic solution was instilled twice a dally into the contralateral eye. They reported that ocular itching was suppressed in both treatment groups compared with a placebo group at 24 hours, and there was no difference in ocular itching suppression or occurrence of adverse drug reactions between the two groups. Mah et al conducted a randomized placebo controlled study using a CAC study protocol in which 92 subjects were distributed into 4 groups: olopatadine hydrochloride vs placebo, epinastine hydrochloride vs placebo, olopatadine 
hydrochloride vs epinastine hydrochloride, and placebo vs placebo (Mah et al 2007). Olopatadine hydrochloride $0.2 \%$ ophthalmic solution significantly suppressed ocular itching by 5-7 minutes after antigen challenge compared with epinastine hydrochloride $0.05 \%$ ophthalmic solution ( $p=0.024$ and $p=0.003$, respectively), and its effects on conjunctival hyperemia and comfort upon instillation were more favorable as well $(p=0.003)$. A placebo-controlled study has also demonstrated the safety profile of olopatadine hydrochloride $0.2 \%$ ophthalmic solution in children and adolescents (Lichtenstein et al 2007). Although olopatadine hydrochloride $0.2 \%$ eye drops cannot be used clinically in Japan, physicians will be attracted to the drug in the near future especially because of its improved compliance.

\section{Possibility of future optional use of olopatadine eye drops}

In Japan among pollinosis patients, treatment for nasal symptoms has been relatively prioritized, while ocular symptoms have not been given sufficient importance (Kakutani et al 2005). Berger et al conducted a prospective multicenter crossover study that involved 200 patients with allergic rhinitis. Of these, $90.5 \%$ had some concurrent ocular symptoms, and ocular treatment with olopatadine hydrochloride $0.1 \%$ eye drops combined with nasal treatment improved ocular allergic symptoms and their QOL compared with the nasal treatment only (Berger et al 2005). From the results in CAC studies, it may be possible that olopatadine hydrochloride $0.1 \%$ or $0.2 \%$ ophthalmic solution has an important role if used for these multi-organ cases by resolving ocular symptoms promptly and potently. Interestingly, it has been reported that olopatadine $0.2 \%$ ophthalmic solution, relative to placebo, significantly reduced the frequency of pollen effects on sneezing and itchy nose, and reduced the severity of pollen effects on sneezing, itchy nose, and runny nose, indicating its additional effect on nasal allergy (Abelson et al 2005).

For patients with a more severe spectrum of ACD, such as VKC and AKC, immunosuppressive eye drops (cyclosporine or tacrolimus) have been introduced for topical treatment in Japan. It has been reported that 2 months' treatment with olopatadine hydrochloride $0.1 \%$ relieves the signs and symptoms of VKC (Corum et al 2005). Also, it reduces the number of goblet cells, which, in turn, decreases the amount of mucus discharge in VKC during treatment. Although similar reports are limited at present, this research suggests that olopatadine $0.1 \%$ eye drops may become an important option in the treatment of VKC and AKC.

\section{Conclusions}

Olopatadine hydrochloride ophthalmic solution has an excellent, strong, and safe anti-allergic effect in vitro and it provides superior clinical effectiveness in patients with AC compared with other histamine antagonistic ophthalmic solutions or non-steroidal anti-inflammatory eye drops. Also, ACD patients feel significantly less discomfort upon instillation. Although further clinical evaluation is necessary, olopatadine hydrochloride eye drops has the potential for simultaneous use with nasal drugs for pollinosis, and also may be used in patients with more severe conditions, such as VKC or AKC, in the near future.

\section{Disclosures}

The author has no conflicts of interest to disclose.

\section{References}

Abelson MB, Chambers WA, Smith LM. 1990. Conjunctival allergen challenge. A clinical approach to studying allergic conjunctivitis. Arch Ophthalmol, 108:84-8.

Abelson MB, Greiner JV. 2004. Comparative efficacy of olopatadine $0.1 \%$ ophthalmic solution versus levocabastine $0.05 \%$ ophthalmic suspension using the conjunctival allergen challenge model. Curr Med Res Opin, 20:1953-8.

Abelson MB, Gomes PJ, Vogelson CT, et al. 2005. Effects of a new formulation of olopatadine ophthalmic solution on nasal symptoms relative to placebo in two studies involving subjects with allergic conjunctivitis or rhinoconjunctivitis. Curr Med Res Opin, 21:683-91.

Abelson MB, Spangler DL, Epstein AB, et al. 2007. Efficacy of once-daily olopatadine $0.2 \%$ ophthalmic solution compared to twice-daily olopatadine $0.1 \%$ ophthalmic solution for the treatment of ocular itching induced by conjunctival allergen challenge. Curr Eye Res, 32:1017-22.

Aguilar AJ. 2000. Comparative study of clinical efficacy and tolerance in seasonal allergic conjunctivitis management with $0.1 \%$ olopatadine hydrochloride versus $0.05 \%$ ketotifen fumarate. Acta Ophthalmol Scand Suppl, 230:52-5.

Artal MN, Luna JD, Discepola M. 2000. A forced choice comfort study of olopatadine hydrochloride $0.1 \%$ versus ketotifen fumarate $0.05 \%$. Acta Ophthalmol Scand Suppl, 230:64-5.

Avunduk AM, Tekelioglu Y, Turk A, et al. 2005. Comparison of the effects of ketotifen fumarate $0.025 \%$ and olopatadine $\mathrm{HCl} 0.1 \%$ ophthalmic solutions in seasonal allergic conjunctivities: a 30-day, randomized, double-masked, artificial tear substitute-controlled trial. Clin Ther, 27:1392-402.

Belfort R, Marbeck P, Hsu CC, et al. 2000. Epidemiological study of 134 subjects with allergic conjunctivitis. Acta Ophthalmol Scand, 78:38-40.

Berdy GJ, Spangler DL, Bensch G, et al. 2000. A comparison of the relative efficacy and clinical performance of olopatadine hydrochloride $0.1 \%$ ophthalmic solution and ketotifen fumarate $0.025 \%$ ophthalmic solution in the conjunctival antigen challenge model. Clin Ther, 22:826-33.

Berger W, Abelson MB, Gomes PJ, et al. 2005. Effects of adjuvant therapy with $0.1 \%$ olopatadine hydrochloride ophthalmic solution on quality of life in patients with allergic rhinitis using systemic or nasal therapy. Ann Allergy Asthma Immunol, 95:361-71.

Brockman HL, Momsen MM, Knudtson JR, et al. 2003. Interactions of olopatadine and selected antihistamines with model and natural membranes. Ocul Immunol Inflamm, 11:247-68.

Calonge M. 1999. Classification of ocular atopic/allergic disorders and conditions: an unsolved problem. Acta Ophthalmol Scand, 77:10-3. 
Cook EB, Stahl JL, Barney NP, et al. 2000. Olopatadine inhibits TNF alpha release from human conjunctival mast cells. Ann Allergy Asthma Immunol, 84:504-8.

Corum I, Yeniad B, Bilgin LK, et al. 2005. Efficiency of olopatadine hydrochloride $0.1 \%$ in the treatment of vernal keratoconjunctivitis and goblet cell density. J Ocul Pharmacol Ther, 21:400-5.

Ganz M, Koll E, Gausche J, et al. 2003. Ketotifen fumarate and olopatadine hydrochloride in the treatment of allergic conjunctivitis: a real-world comparison of efficacy and ocular comfort. Adv Ther, 20:79-91.

Katelaris CH, Ciprandi G, Missotten L; International Olopatadine Study Group. 2002. A comparison of the efficacy and tolerability of olopatadine hydrochloride $0.1 \%$ ophthalmic solution and cromolyn sodium $2 \%$ ophthalmic solution in seasonal allergic conjunctivitis. Clin Ther, 24:1561-75.

Knight A. 1994. The role of levocabastine in the treatment of allergic rhinoconjunctivitis. Br J Clin Pract, 48:139-43.

Lanier BQ, Finegold I, D’Arienzo P, et al. 2004. Clinical efficacy of olopatadine vs epinastine ophthalmic solution in the conjunctival allergen challenge model. Curr Med Res Opin, 20:1227-33.

Lichtenstein SJ, Pasquine TA, Edwards MR, et al. 2007. Safety and tolerability of olopatadine $0.2 \%$ in children and adolescents. $J$ Ocul Pharmacol Ther, 23:366-71.

Mah FS, Rosenwasser LJ, Townsend WD, et al. 2007. Efficacy and comfort of olopatadine $0.2 \%$ versus epinastine $0.05 \%$ ophthalmic solution for treating itching and redness induced by conjunctival allergen challenge. Curr Med Res Opin, May 18; [Epub ahead of print].

Ohmori K, Hayashi K, Kaise T, et al. 2002. Pharmacological, pharmacokinetic and clinical properties of olopatadine hydrochloride, a new antiallergic drug. Jpn J Pharmacol, 88:379-97.

Ohno S, Uchio E, Takamura E, et al. 2007. Efficacy and comfort of 0.1\% olopatadine hydrochloride ophthalmic solution for allergic conjunctivitis in the Japanese. Comparison with $0.025 \%$ levocabastine hydrochloride ophthalmic solution. Rinsho Ganka (Journal of Clinical Ophthalmology) [in Japanese], 61:251-5.

Ono SJ, Abelson MB. 2005. Allergic conjunctivitis: Update on pathophysiology and prospects for future treatment. J Allergy Clin Immunol, 115:118-22.
Saiga T. 2006. Clinical evaluation of olopatadine hydrochloride ophthalmic solution (ALO4943A) in allergic conjunctivitis - Double-masked comparison with ketotifen fumarate ophthalmic solution. Atarashii Ganka (Journal of the Eye) [in Japanese], 23:1337-52.

Saiga T, Fujioka T, Ogawa Y, et al. 2006. Dose-finding study of $0.1 \%$ ALO4943A in allergic conjunctivitis (BID vs QID). Atarashii Ganka (Journal of the Eye) (in Japanese), 23:1325-36.

Saiga T, Mii S, Hayashi K. 2006. Long-term study of 0.1\% ALO4943A in allergic conjunctivitis. Atarashii Ganka (Journal of the Eye) [in Japanese], 23:1501-10.

Sharif NA, Xu SX, Yanni JM. 1996. Olopatadine (AL-4943A): ligand binding and functional studies on a novel, long acting $\mathrm{H} 1$-selective histamine antagonist and anti-allergic agent for use in allergic conjunctivitis. J Ocul Pharmacol Ther, 12:401-7.

Spangler DL, Bensch G, Berdy GJ. 2001. Evaluation of the efficacy of olopatadine hydrochloride $0.1 \%$ ophthalmic solution and azelastine hydrochloride $0.05 \%$ ophthalmic solution in the conjunctival allergen challenge model. Clin Ther, 23:1272-80.

Sumi T, Fukushima A, Sawada K, et al. 2008. Evaluation of $0.1 \%$ olopatadine hydrochloride ophthalmic solution for allergic conjunctivitis. Rinsho Ganka (Journal of Clinical Ophthalmology) [in Japanese], 62:313-7.

Vogelson CT, Abelson MB, Pasquine T, et al. 2004. Preclinical and clinical antiallergic effect of olopatadine $0.2 \%$ solution 24 hours after topical ocular administration. Allergy Asthma Proc, 25:69-75.

Yanni JM, Miller ST, Gamache DA, et al. 1997. Comparative effects of topical ocular anti-allergy drugs on human conjunctival mast cells. Ann Allergy Asthma Immunol, 79:541-5.

Yanni JM, Stephens DJ, Miller ST, et al. 1996. The in vitro and in vivo ocular pharmacology of olopatadine (AL-4943A), an effective anti-allergic/ antihistaminic agent. J Ocul Pharmacol Ther, 12:389-400.

Yanni JM, Weimer LK, Sharif NA, et al. 1999. Inhibition of histamineinduced human conjunctival epithelial cell responses by ocular allergy drugs. Arch Ophthalmol, 117:643-7.

Yaylali V, Demirlenk I, Tatlipinar S, et al. 2003. Comparative study of $0.1 \%$ olopatadine hydrochloride and $0.5 \%$ ketorolac tromethamine in the treatment of seasonal allergic conjunctivitis. Acta Ophthalmol Scand, 81:378-82. 
